\title{
Genetic Factors Determining the Growth of Physarum polycephalum Amoebae in Axenic Medium
}

\author{
By CLARE H. R. MCCULLOUGH, JENNIFER DEE AND \\ JENNIFER L. FOXON \\ Department of Genetics, University of Leicester, Leicester LE1 7RH \\ (Received 14 November 1977; revised 2 February 1978)
}

\begin{abstract}
Evidence is presented that in the true slime mould Physarum polycephalum the ability of the amoebal strain CLd-AXE to grow in axenic medium is determined by mutations in two genes axe $A$ and $a x e B$ and that the axenic growth of RSD4-AXE amoebae is also under genetic control. Mutant amoebal strains are also able to grow on bacterial lawns and the ability to grow in axenic media persists during prolonged culture on bacteria. However, some of the mutant strains grow less well on bacteria than strains of similar genetic background which are unable to grow in axenic media. CLd-AXE has the same nuclear DNA content $(0.59 \mathrm{pg}$ per nucleus) as CLd, the strain from which it was derived. Amoebae able to grow in axenic media were also derived from strains E27 and Lu858 which carry mutations for temperature sensitivity and leucine requirement expressed in the plasmodial phase. Tests in axenic media showed that these mutations were expressed in the amoebal phase. The elucidation of the genetic basis of axenic growth will allow the construction of a range of amoebal strains able to grow in axenic media and this will greatly facilitate the isolation and analysis of mutants in this organism.
\end{abstract}

\section{INTRODUCTION}

For genetic analysis of the true slime mould Physarum polycephalum, amoebal strains are routinely cloned and cultured on lawns of bacteria (Escherichia coli) on agar. Goodman (1972) successfully established one strain (RSD4) in a complex liquid medium. Two strains (RSD4 and CLd) were eventually cultured in simpler semi-defined and fully defined media but only grew well after long periods of adaptation (McCullough \& Dee, 1976). Attempts to transfer other strains from two-membered culture to the semi-defined or defined media repeatedly failed.

The defined axenic media were developed to facilitate the isolation of auxotrophic mutants and the testing of amoebal strains carrying auxotrophic mutations identified in plasmodia. Progress towards both these objectives was obstructed by the long adaptation period required to establish amoebal strains in axenic culture. This paper describes experiments designed to test whether the RSD4 and CLd strains established in axenic media were physiologically or genetically different from RSD4 and CLd amoebae which had been continuously cultured on $E$. coli. The results suggest that the ability of cLd to grow in axenic media is conferred by mutations at two loci and can be transferred to other amoebal strains by simple crosses. Ability to grow in axenic media is genetically determined in the cellular slime mould Dictyostelium discoideum (Williams, Kessin \& Newell, 1974). 


\section{METHODS}

Loci. $m t$, amoebal mating-type: alleles $m t_{1}, m t_{2}$ etc. control heterothallic plasmodium formation (Dee, 1966); allele $m t_{\mathrm{h}}$ confers the ability to form plasmodia within amoebal clones (Wheals, 1970). fus $A$, plasmodial fusion type (Poulter \& Dee, 1968): alleles fus $A 1$ and fus $A 2$ are co-dominant; only plasmodia carrying the same alleles fuse. Haploid plasmodia (e.g. fus $A$ l) fuse with diploid plasmodia homozygous for the same allele (i.e. fus Al/fusAl). leu, plasmodial requirement for leucine: mutant allele leu is recessive (Cooke \& Dee, 1975). $h t s$, plasmodial temperature sensitivity; mutant allele $h t s-l$ is recessive.

Amoebal strains. RSD4 is a heterothallic clone $\left(m t_{1}\right)$ originally described by Haugli (1971) and obtained from him; its plasmodial fusion type is unknown. CLd $\left(m t_{\mathrm{h}} ; f u s A 2\right)$ is a derivative of CL (Wheals, 1970) showing delayed clonal plasmodium formation (Cooke \& Dee, 1975). All $m t_{\mathrm{h}}$ progeny from crosses between CLd and heterothallic strains are delayed in clonal plasmodium formation. Plasmodia derived clonally from CLd amoebae yield $m t_{\mathrm{h}}$ progeny which are not delayed in clonal plasmodium formation. Other strains used were as follows: Lu862 ( $m t_{3} ;$ fusAl) (Anderson \& Dee, 1977); LU858 ( $m t_{2} ;$ fusAl; leu); E27 $\left(m t_{\mathrm{h}} ; h t s-1\right)$ (Gingold et al., 1976); LU688 (mt $;$ fus $A 1)$ (Cooke \& Dee, 1975); LU853 ( $m t_{1} ; f_{u s A 2}$ ).

Axenic media for culture of amoebae. Axenic media were as previously described (McCullough \& Dee, 1976) except for the modifications indicated. ASDM (amoebal semi-defined medium): Oxoid bacteriological peptone, $1 \%(\mathrm{w} / \mathrm{v})$; glucose, $1 \%(\mathrm{w} / \mathrm{v})$; other components as in BTC (Henney \& Henney, 1968) but omitting casein hydrolysate. SDM (semi-defined medium): as used for plasmodia (Dee \& Poulter, 1970). MADM-13 (modified amoebal defined medium containing 13 amino acids): glucose, $10 \mathrm{~g} \mathrm{l}^{-1}$; L-alanine, 54.6 $\mathrm{mg} \mathrm{l}^{-1}$; L-arginine. $\mathrm{HCl}$, 40.8 $\mathrm{mg} \mathrm{1}^{-1}$; glycine, $30.6 \mathrm{mg} \mathrm{1}^{-1}$; L-leucine, $70.8 \mathrm{mg} \mathrm{l}^{-1}$; L-isoleucine, $11.8 \mathrm{mg} \mathrm{1}^{-1}$; L-valine, $15 \cdot 2 \mathrm{mg} \mathrm{l}^{-1}$; L-lysine, $10.6 \mathrm{mg} \mathrm{l}^{-1}$; other components as in ADM-13 (McCullough \& Dee, 1976).

Methods for culturing and sampling amoebae in axenic media were as described by McCullough \& Dee (1976). For all growth tests, $100 \times 16 \mathrm{~mm}$ plastic tubes were used containing 1 to $2 \mathrm{ml}$ medium and incubated horizontally in stationary racks. The first culture of a strain in a particular medium is referred to as the 'initial' culture and subsequent cultures as 'subcultures'.

Culture of amoebae on agar with E. coli. Amoebae were cultured on liver infusion agar [Oxoid liver infusion powder, $1 \mathrm{~g} \mathrm{l}^{-1}$; agar, $20 \mathrm{~g} \mathrm{1}^{-1}$ ] with living $E$. coli (LIA cultures) or on a lawn of formalin-killed $E$. coli (Haugli, 1971) (FK/LIA cultures). For both types of culture, methods of subculturing and cloning were as described by Anderson \& Dee (1977).

Measurements of plating efficiency and amoebal plaque size on agar. For strains growing in axenic medium, the number of cells in a sample of the culture was estimated using a haemocytometer and the culture was diluted with the same type of medium to give appropriate cell numbers. For amoebae growing on $E$. coli, each culture was washed off the plate by flooding it with $2 \mathrm{ml}$ axenic medium and gently scraping with a glass spreader. The number of cells in this suspension was estimated using a haemocytometer and appropriate dilutions were made in axenic medium. A known number of amoebae (between 60 and 300) was then plated on either LIA or FK/LIA. The number of amoebal plaques per plate was counted after incubation and the mean for each strain on each substrate was expressed as a percentage of the estimated number of amoebae plated (plating efficiency). To measure plaque size, a straight line $(9 \mathrm{~cm})$ was scratched across the back of each plastic plate and the diameters of all plaques (approximately 5 to 10) on this line were measured at intervals during the incubation period.

Crossing and plasmodium formation. The methods described by Anderson \& Dee (1977) were used for crossing amoebal strains, culturing plasmodia, obtaining spores and isolating progeny clones of amoebae. In crosses between $m t_{\mathrm{h}}$ amoebae (CLd) and heterothallic strains, hybrid plasmodia were identified by means of the plasmodial fusion system (see Results). To test for plasmodium formation within clones and to obtain plasmodia from $m t_{\mathrm{h}}$ strains, amoebae were inoculated into a $0.2 \mathrm{ml}$ drop of $E$. coli suspension (approximately $\left.10^{10} \mathrm{ml}^{-1}\right)$ on dilute SDM agar [6.25 $\mathrm{ml} \mathrm{SDM} \mathrm{per} 100 \mathrm{ml} 2 \%(\mathrm{w} / \mathrm{v})$ agar].

Growth tests of progeny strains in axenic media. An amoebal strain was grown on FK/LIA until plaques were almost confluent. The plate was then flooded with $2 \mathrm{ml}$ axenic medium (SDM or ASDM) and the amoebae were gently scraped off with a glass spreader. Approximately $1 \mathrm{ml}$ was removed with a pipette and transferred to an empty culture tube. This usually yielded a culture of more than $5 \times 10^{5} \mathrm{cells}^{-1}$. This initial culture was incubated for a week and then subcultured by transferring $1 \mathrm{ml}$ to a tube containing $1 \mathrm{ml}$ fresh medium. This subculture was incubated for a week and then a second subculture was made; after another week's incubation, the cells were counted. Cultures containing more than $4 \times 10^{6} \mathrm{cells}^{\mathrm{ml}} \mathrm{I}^{-1}$ at the end of this 3-week test period were scored as growing in axenic medium. Tests were done in ASDM or SDM as specified in Results.

Isolation of nuclei and estimation of DNA content. Plasmodial nuclei were isolated in G2 phase according to the method of Mohberg \& Rusch (1971) and amoebal nuclei were isolated by a modification of this method (Mohberg, 1977). Duck erythrocyte nuclei were isolated by the method described for isolating chicken 
erythrocyte nuclei (Mohberg, 1977). Suspensions of nuclei were frozen in liquid nitrogen and stored at $-20^{\circ} \mathrm{C}$. Isolated nuclei were stained with leuco-basic fuchsin as described by Cooke \& Dee (1974). Stain intensity in individual nuclei was measured with a Vickers M85 scanning microdensitometer (Vickers Instruments, York YO3 7SD) set with slit width 25, wavelength 55 and spot size 2 . The mean scanning density of 25 duck erythrocyte nuclei, stained in the same batch as the $P$. polycephalum nuclei, was taken to represent a value of $2.7 \mathrm{pg}$ DNA (Mirsky \& Ris, 1951).

\section{RESULTS}

\section{Tests of RSD4 and CLd in axenic medium after several subcultures on bacteria}

To test whether ability to grow in axenic medium was a reversible change and therefore possibly a physiological adaptation, amoebae of strain RSD4 which had been grown axenically for at least 5 years were transferred from axenic medium (ASDM) to lawns of formalinkilled $E$. coli on liver infusion agar (FK/LIA plates) and subcultured seven times. This population is referred to as RSD4-AXE. RSD4 amoebae which had never been grown in axenic medium were transferred from lawns of live bacteria on LIA to FK/LIA plates containing streptomycin $\left(0 \cdot 31 \mathrm{~g}^{-1}\right)$ and subcultured twice to rid them of live bacteria. This population was designated RSD4-BACT. A plate of each population was used to inoculate an ASDM liquid culture and the growth of the amoebae was followed in this initial culture and through two subcultures. RSD4-AXE grew at a similar rate in all three cultures; RSD4-BACT grew well initially, but very little growth occurred in the first subculture and none at all in the second (Fig. 1). This result indicated that the 'adaptation' to axenic growth had survived through approximately 20 doublings on FK/LIA plates. Populations of CLd amoebae from axenic culture in ASDM and from culture on E. coli, designated CLd-AXE and CLd-BACT respectively, were compared in the same way and a similar result was obtained.

\section{Tests in axenic medium of clonal progeny of CLd-AXE}

If the CLd-AXE amoebae were able to grow in axenic medium as a result of a genetic change, this ability should be inherited through the apogamic life-cycle. A plasmodium was obtained from CLd-AXE amoebae and this was induced to form spores (CLd-AXE spores). It was known that the amoebal progeny of CLd-AXE would no longer show delayed plasmodium formation (Cooke \& Dee, 1975) and previous experiments on strain CL had shown that 'non-delayed' amoebae readily formed plasmodia in axenic medium. Therefore, before the growth characteristics of the amoebal progeny could be followed, it was necessary to obtain amoebae which were delayed in plasmodium formation from the CLd-AXE spores; the enrichment method of Cooke \& Dee (1975) was used to select such amoebae. CLd-AXE spores were plated on FK/LIA and incubated until plasmodia appeared in the amoebal plaques. The plates were then refrigerated $\left(4^{\circ} \mathrm{C}\right)$ to kill the plasmodia. After $24 \mathrm{~h}$, the plates were flooded with $2 \mathrm{ml}$ water and gently scraped with a glass spreader. The resulting suspension was replated in $0.1 \mathrm{ml}$ portions on FK/LIA and three amoebal clones were obtained which showed very marked delay in plasmodium formation. When amoebae pooled from these clones (CLd-AXEprogeny), grown on FK/LIA plates, were inoculated into liquid SDM, growth occurred immediately and continued through at least six subcultures. The doubling time (1.5 to $2 \mathrm{~d}$ ) was similar to that of CLd-AXE.

\section{Analysis of progeny of the cross CLd-AXE $\times$ LU862}

To investigate the genetic basis of axenic growth, a cross was set up between CLd-AXE amoebae taken from axenic cultures and a strain which could not grow in axenic medium, LU862. The progeny of a hybrid plasmodium (identified as fusAl/fus $A 2$ fusion type) were analysed for mating-type and for ability to grow in axenic medium (SDM) after a standard 3-week test period (see Methods). This procedure was adopted on the basis of the result in Fig. 1 which showed that differences between strains in their ability to grow in axenic medium were not apparent until the second subculture. 


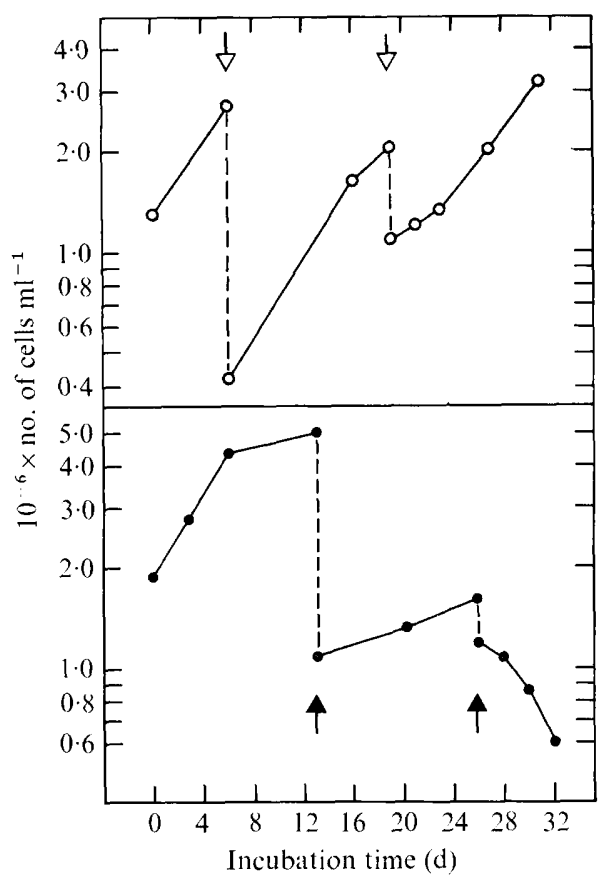

Fig. 1

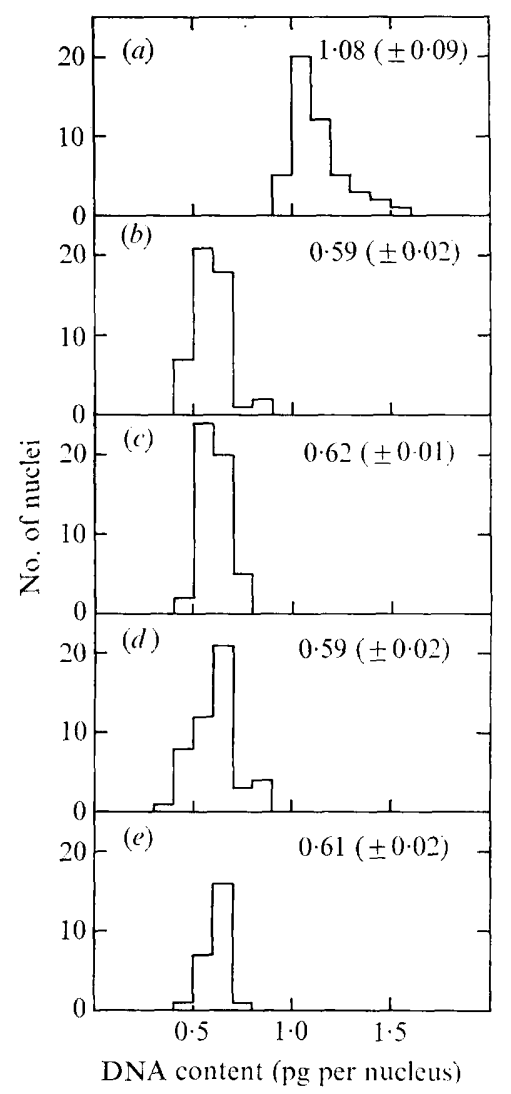

Fig. 2

Fig. 1. Growth of RSD4 amoebae in ASDM; the arrows indicate the times when the first and second subcultures were made. $\bigcirc$, RSD4-AXE amoebae inoculated after seven transfers on FK/LIA; $\bigcirc$, RSD4-BACT amoebae inoculated from FK/LIA.

Fig. 2. DNA contents of nuclei isolated from: $(a)$ a diploid plasmodium (LU688 $\times$ LU853); (b) CLdBACT plasmodium; $(c)$ CLd-AXE plasmodium; $(d)$ CLd-AXE amoebae; $(e)$ CLd-AXE-progeny amoebae. Microdensitometer readings were made on 50 Feulgen-stained nuclei for each strain (only 25 for CLd-AXE-progeny) and the DNA content of each nucleus was calculated using isolated duck erythrocyte nuclei as an internal standard (see Methods). Mean DNA contents (pg per nucleus) are given for each sample.

Of 60 progeny clones tested, 17 grew in axenic medium (axenic growers), the mean cell number being $5.8 \times 10^{6} \mathrm{ml}^{-1}$ after the test period; the remaining 43 clones had a mean cell number of $5.1 \times 10^{5} \mathrm{ml}^{-1}$, which did not represent an increase over the initial inoculum, and were therefore classified as non-growers (Table 1). The axenic growers and non-growers differed markedly in appearance; the axenic growers consisted of relatively large cells with characteristic amoeboid shape and obvious cell contents, whereas cultures of non-growers contained small, rounded cells, a great deal of cell debris and many 'ghosts', presumably empty cyst walls.

The ratio of growers to non-growers was not significantly different from 1:3 ( $\chi^{2}$ test; $P=0.65$ ), which is the expected ratio if the ability to grow in axenic medium involves two unlinked mutations (axeAl and axeBI) both of which are necessary for growth. The ratio of $m t_{\mathrm{h}}: m t_{3}$ did not deviate significantly at the $5 \%$ level from $1: 1$ among either growers or non-growers (Table 1), indicating that $m t$ was not linked to axe $A$ or axeB. A sample of the $m t_{\mathrm{h}}$ progeny was scored for plasmodial fusion type and fus $A 1$ and fus $A 2$ were found among 


\section{Table 1. Analysis of progeny of the cross CLd-AXE $\times$ LU862}

Strain CLd-AXE $\left(m t_{\mathrm{h}} ; \mathrm{fusA2}\right.$; able to grow in axenic medium) was crossed with strain LU862 $\left(m t_{3}\right.$; fus $A 1$; not able to grow in axenic medium). After cloning on FK/LIA, progeny strains were inoculated into axenic medium (SDM) and scored for growth at the end of a 3-week test period (see Methods).

\begin{tabular}{cccc} 
& \multicolumn{3}{c}{ Number of progeny } \\
\cline { 2 - 4 } Mating-type & $\begin{array}{c}\text { Axenic } \\
\text { growers }\end{array}$ & Non-growers & Total \\
$m t_{\mathrm{h}}$ & 6 & 21 & 27 \\
$m t_{3}$ & 11 & 22 & 33 \\
Total & 17 & 43 &
\end{tabular}

Table 2. Analysis of progeny of the cross CLd-AXE $\times$ RSD4-AXE

Strain CLd-AXE ( $m t_{\mathrm{h}}$; fusA2; able to grow in axenic medium) was crossed with strain RSD4-AXE ( $m t_{1}$; fusion type unknown; able to grow in axenic medium). Progeny strains were scored for ability to grow in axenic medium (SDM) and mating-type (see Table 1 and Methods).

\begin{tabular}{cccc} 
Mating-type & \multicolumn{3}{c}{ Number of progeny } \\
$\overbrace{\begin{array}{c}\text { Axenic } \\
\text { growers }\end{array}}$ & Non-growers & Total \\
$m t_{\mathrm{n}}$ & 11 & 8 & 19 \\
Unknown* & 7 & 10 & 17 \\
Total & 7 & 6 & 13
\end{tabular}

* These progeny could not be scored for mating-type because of contamination on the test plates.

both axenic growers and non-growers, showing that recombination had occurred between fus $A$ and ability to grow in axenic medium.

The 17 progeny clones which had been scored as growing in SDM were also tested for their ability to grow in fully defined medium (MADM-13). At the end of the 3-week test period, each of the 17 cultures contained more than $4 \times 10^{6}$ cells $\mathrm{ml}^{-1}$ indicating that growth had occurred.

\section{Analysis of progeny of the cross CLd-AXE $\times$ RSD4-AXE}

To investigate whether the same mutations were responsible for axenic growth in RSD4-AXE as in CLd-AXE, cells taken from axenic cultures were used to set up a cross between the two strains. Crossed plasmodia were identified as those failing to fuse with a fus $A 2$ tester strain. Progeny of a hybrid plasmodium were classified for ability to grow in axenic medium (SDM) and for mating-type (Table 2). Of the 49 progeny clones tested, 25 grew in axenic medium, the mean cell number being $6.2 \times 10^{6} \mathrm{ml}^{-1}$ after the test period; the remaining 24 clones failed to grow and had a mean cell number of $1.3 \times 10^{6} \mathrm{ml}^{-1}$.

The segregation of growers and non-growers was evidence that the parent strains carried different mutations for axenic growth; if they had carried identical mutations, non-growers would not have been expected among the progeny. A genetic model which is consistent with the results is presented in the Discussion.

The segregation of mating-type $\left(m t_{\mathrm{h}}, m t_{1}\right)$ did not deviate from the expectation of $1: 1$ among the total progeny ( $\chi^{2}$ test; $P=0 \cdot 7$ ) or among either the axenic growers or nongrowers (Table 2).

\section{DNA contents of CLd-AXE and CLd-BACT}

To investigate their DNA content, nuclei were isolated from amoebae of CLd-AXE and CLd-AXE-progeny in exponential growth in axenic medium and from a CLd-AXE plasmodium in G2 phase. As controls, G2 phase nuclei were isolated from a plasmodium assumed to be 


\title{
Table 3. Comparative growth of axenic growing and non-growing amoebae on agar media
}

\begin{abstract}
Mean plaque diameter was calculated from measurements after $8 \mathrm{~d}$ incubation of 10 to 20 plaques on each of three replicate plates for each strain on each medium. Plating efficiency was the number of plaques per plate expressed as a percentage of the estimated number of cells plated. Counts were made initially when plaques were clearly visible but additional counts were made until plaques were becoming confluent. Time for plaque formation was the incubation period necessary for the formation of plaques $>0.5 \mathrm{~mm}$ diameter.
\end{abstract}

\begin{tabular}{|c|c|c|c|c|}
\hline Strain & Agar medium & $\begin{array}{l}\text { Mean plaque } \\
\text { diam. (mm) }\end{array}$ & $\begin{array}{c}\text { Plating efficiency } \\
(\%)\end{array}$ & $\begin{array}{l}\text { Time fo } \\
\text { plaque } f \\
\text { mation }\end{array}$ \\
\hline \multicolumn{5}{|c|}{ (a) CLd-AXE and CLd-BACT plated after three subcultures on FK/LIA } \\
\hline CLd-BACT & $\begin{array}{l}\text { LIA } \\
\text { FK/LIA }\end{array}$ & $\begin{array}{l}3.75 \pm 0.73 \\
1.75 \pm 0.13\end{array}$ & $\begin{array}{l}177 \pm 31 \\
127 \pm 26\end{array}$ & $\begin{array}{r}<4 \\
6\end{array}$ \\
\hline CLd-AXE & $\begin{array}{l}\text { LIA } \\
\text { FK/LIA }\end{array}$ & $\begin{array}{l}1.84 \pm 0.12 \\
<0.5\end{array}$ & $\begin{array}{c}109 \pm 11 \\
18 \pm 5\end{array}$ & $\begin{array}{l}5 \\
8\end{array}$ \\
\hline
\end{tabular}

(b) CLd-AXE and LU310 plated directly from axenic medium compared with CLd-BACT

$\begin{array}{llllr}\text { CLd-BACT } & \text { LIA } & 2 \cdot 73 \pm 0 \cdot 23 & 160 \pm 20 & <4 \\ & \text { FK/LIA } & 1 \cdot 13 \pm 0 \cdot 05 & 118 \pm 27 & 6 \\ \text { CLd-AXE } & \text { LIA } & 2 \cdot 30 \pm 0 \cdot 16 & 50 \pm 3 & 6 \\ & \text { FK/LIA } & <0 \cdot 5 & 13 \pm 1 & 11 \\ \text { LU310 } & \text { LIA } & 3 \cdot 13 \pm 0 \cdot 52 & 61 \pm 11 & 6 \\ & \text { FK/LIA } & 1 \cdot 19 \pm 0 \cdot 20 & 64 \pm 5 & 8\end{array}$

haploid (CLd-BACT) and from the diploid plasmodium LU688 $\times$ LU853. The DNA content of the isolated nuclei was estimated as described in Methods, using duck erythrocyte nuclei as an internal standard. CLd-AXE and CLd-AXE-progeny had similar DNA contents (Fig. 2) to the CLd-BACT control which had a mean content $(0.59 \pm 0.02 \mathrm{pg}$ DNA per nucleus) close to that previously reported for CL (Mohberg, 1977; Anderson \& Dee, 1977).

\section{Comparative growth tests of $\mathrm{CLd}-\mathrm{AXE}$ and $\mathrm{CLd}-\mathrm{BACT}$}

Amoebal growth on agar. CLd-AXE and CLd-BACT amoebae were subcultured three times on FK/LIA and then plated on LIA and FK/LIA to compare plaque size and plating efficiency (see Methods). Plaques of measurable diameter $(>0.5 \mathrm{~mm}$ ) took longer to appear for CLdAXE than for CLd-BACT. Plaque diameter, measured at $2 \mathrm{~d}$ intervals, was always smaller for CLd-AXE than for CLd-BACT on both LIA and FK/LIA; measurements after $8 \mathrm{~d}$ are shown in Table 3(a). For each strain, the mean plaque diameter was smaller on FK/LIA than on LIA. Plating efficiency was near $100 \%$ except for CLd-AXE on FK/LIA, where it was only $18 \%$ (Table $3 a$ ). The progeny amoebae clonally derived from CLd-AXE (CLd-AXE-progeny) gave results similar to those of CLd-AXE.

Plasmodial growth on agar. Plasmodia derived from CLd-AXE and CLd-BACT amoebae were inoculated in triplicate on plates of SDM agar, using inoculum blocks of $3 \times 3 \mathrm{~mm}$. The CLd-AXE plasmodium grew more slowly, taking at least $4 \mathrm{~d}$ to cover the plates, while all three subcultures of the CLd-BACT plasmodium were fully grown after only $3 \mathrm{~d}$. However, when tested on minimal defined agar medium (DM-1; Dee, Wheals \& Holt, 1973), both plasmodia grew, indicating that CLd-AXE had no additional requirements.

\section{Comparative growth tests of CLd-AXE and LU310}

To investigate whether the poor amoebal growth and low plating efficiency on bacteria shown by CLd-AXE and CLd-AXE-progeny were characteristic of other strains able to grow in axenic medium, one of the axenic growers among the progeny of CLd-AXE $\times$ LU862 (designated LU310) was compared with CLd-AXE.

Amoebal growth on agar. CLd-AXE and LU310 were plated directly from liquid SDM cultures on to LIA and FK/LIA and compared with CLd-BACT as a control. On FK/LIA, 


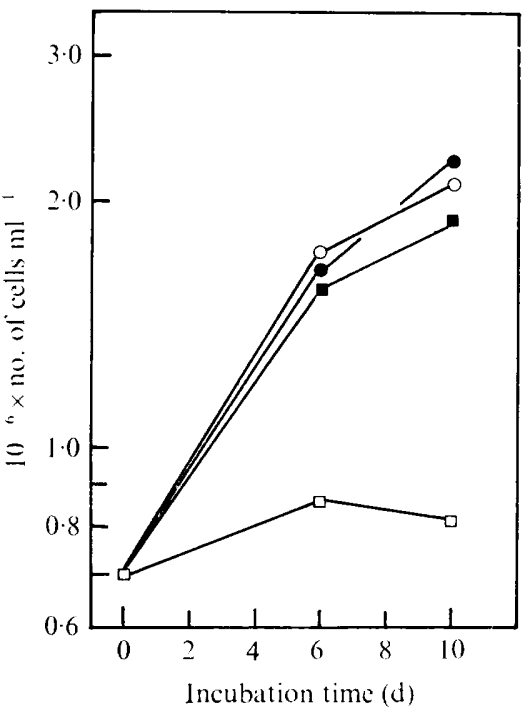

Fig. 3

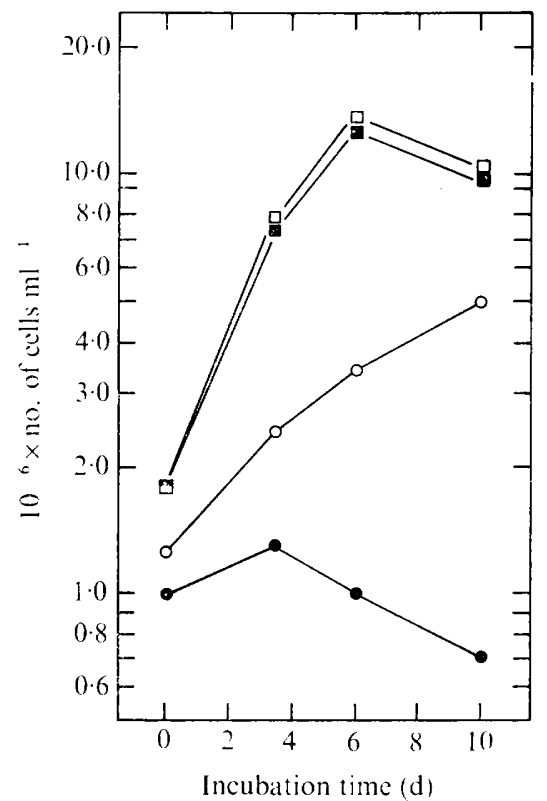

Fig. 4

Fig. 3. Test for leucine requirement of LU858 and CLd-AXE amoebae. Cells were transferred from MADM-13 to MADM-13 and MADM-13 without leucine and subcultured twice in these media. Growth during the second subculture is shown for: $\mathbf{0}$, CLd-AXE in MADM-13; $\mathbf{n}$, CLd-AXE in MADM-13 without leucine; $\bigcirc$, LU858 in MADM-13; $\square$, LU858 in MADM-13 without leucine.

Fig. 4. Test for temperature sensitivity of amoebae of CLd-AXE and E27. Cells were transferred from SDM at $26^{\circ} \mathrm{C}$ to SDM at 26 and $31^{\circ} \mathrm{C}$. Growth of these initial cultures is shown for: $\square$, CLd-AXE at $26^{\circ} \mathrm{C} ; \mathrm{O}$, CLd-AXE at $31{ }^{\circ} \mathrm{C} ; \mathbf{\square}, \mathrm{E} 27$ at $26^{\circ} \mathrm{C} ; \boldsymbol{O}, \mathrm{E} 27$ at $31^{\circ} \mathrm{C}$.

measurable plaques of LU310 appeared sooner than those of CLd-AXE and at all stages plaque diameter was similar to that of the CLd-BACT control (Table $3 b$ ). On LIA, differences in mean plaque diameter between the strains were less marked and plaques of all strains were larger than on FK/LIA. As before, the plating efficiency of CLd-AXE on FK/LIA was very low $(13 \%)$, but for LU310 the plating efficiency was more than $60 \%$ on both media (Table $3 b$ ).

Amoebal growth in axenic medium. The growth of LU310 and CLd-AXE was compared in SDM and MADM-13. In both media, LU310 grew slightly more slowly than CLd-AXE.

\section{Tests of amoebal strains carrying mutations identified in plasmodia}

During the course of the genetic analysis described above, attempts were made to establish in axenic medium amoebal strains carrying mutations which had been identified in plasmodia. After many subcultures in which very little growth could be detected, the growth rate of two strains (LU858 and E27) improved sufficiently to allow tests of their mutant characteristics. The basis of ability to grow in axenic medium in these strains has not yet been investigated.

Strain Lu858. The recessive allele leu determines a specific requirement for leucine in plasmodia but it has not previously been possible to test whether this mutation is expressed in amoebae. Amoebae of LU858 $\left(\mathrm{mt}_{2} ; \mathrm{leu}\right)$ which had acquired the ability to grow in axenic medium (SDM) were transferred to MADM-13 and MADM-13 without leucine. The growth of LU858 and the CLd-AXE control in the second subculture in both media is illustrated in Fig. 3. While CLd-AXE grew equally well in both media, LU858 showed very little growth in the absence of leucine and even before this subculture the cells were abnormally large in this 
medium. The results show that leucine is not an absolute requirement of CLd-AXE amoebae and that the mutation leu is expressed in Lu858 amoebae.

Strain E27. Plasmodia of the mutant strain E27 $\left(m t_{\mathrm{h}} ; h t s-1\right)$ are unable to grow at $31^{\circ} \mathrm{C}$ which is a permissive temperature for CLd plasmodia. $\mathrm{E} 27$ amoebae which had acquired the ability to grow in liquid SDM at $26^{\circ} \mathrm{C}$ were inoculated into SDM which had been held at $31{ }^{\circ} \mathrm{C}$ overnight and into SDM at $26^{\circ} \mathrm{C}$. Cultures of CLd-AXE amoebae were set up in the same way as controls. At $31^{\circ} \mathrm{C}$, E27 amoebae grew very poorly compared with either the same strain at $26^{\circ} \mathrm{C}$ or CLd-AXE at $31^{\circ} \mathrm{C}$ (Fig. 4).

\section{DISCUSSION}

Results of the cross CLd-AXE $\times$ LU862 were consistent with the hypothesis that strain CLd-AXE carries two unlinked mutations, axe $A 1$ and axeBl, both of which are necessary for growth in axenic media. These mutations recombined with $m t$ and fus $A$. During prolonged axenic culture the growth rate of CLd-AXE has increased, so it is possible that the two mutations which initially allowed axenic growth have been followed by modifying mutations. The slightly reduced growth in axenic medium of the progeny strain LU310 also suggests the presence of modifiers in the parent CLd-AXE. However, if these additional mutations were essential, the proportion of axenic growers among progeny of the cross CLd-AXE $\times$ LU862 would have been much lower than that observed.

The observed segregation of 25 growers to 24 non-growers in the cross between two axenic growers (CLd-AXE $\times$ RSD4-AXE) suggests two alternative genetic models. With model $(a)$, RSD4-AXE carries one mutation (axe C1) in a gene unlinked to axe $A$ and $a x e B$. Assuming that the progeny which can grow in axenic medium are those carrying axe Al axeB1 (regardless of their genotype at axe $C$ ) and those carrying axe $C 1$ (regardless of their genotype at axe $A$ and $a x e B)$, the expected ratio of axenic growers: non-growers is $5: 3$. With model $(b)$, RSD4-AXE carries two mutations axe $C 1$ and axeD1, unlinked to each other and to axe $A$ and axeB. Assuming that the progeny which can grow in axenic medium are those carrying either axe $A 1$ axeBl or axeCl axeDI (regardless of their genotype at the other two axe loci) the expected ratio of growers: non-growers is 7:9. The data are consistent with both models but the probability of obtaining the observed segregation ratio is greater for $(b)(P=0 \cdot 3)$ than for (a) $(P=0 \cdot 1)$.

In addition to RSD4 and CLd, the strains E27 and LU858 have now been grown axenically. RSD4 and CLd are derived from different natural isolates whereas E27 and Lu858, like most other strains now used in $P$. polycephalum genetics, are isogenic with CL and CLd. Mutations have presumably occurred in E27 and Lu858 enabling them to grow in axenic media and it will be interesting to determine whether these strains, unlike RSD4-AXE, carry the same mutations as CLd-AXE.

It seems unlikely that mutations have occurred which enable the amoebae to synthesize a nutrient formerly supplied by the bacteria. Since the mutant amoebae can be grown in unmodified plasmodial medium, it is possible that the mutations are of a regulatory nature allowing the expression of genes normally expressed only in plasmodia. Another possibility is that one or both of the mutations affects the surface properties of amoebae so that they can survive in the hypotonic culture media. Whatever their nature, the axe mutations do not prevent amoebae from growing on bacteria. The functions of the two genes known to control axenic growth in the cellular slime mould $D$. discoideum are also unknown.

While amoebal strains used for genetic analysis are normally stored at $4{ }^{\circ} \mathrm{C}$ and subcultured infrequently, CLd-AXE amoebae have been subcultured regularly for several years. Large increases in nuclear DNA content associated with senescence have been reported during prolonged culture of amoebae on bacterial lawns (Adler \& Holt, 1974) and of plasmodia on agar media (McCullough et al., 1973). The CLd-AXE plasmodium also showed poor growth on agar media similar to that shown by senescent plasmodia. However, the 
nuclear DNA contents of CLd-AXE amoebae, a CLd-AXE plasmodium and the clonal progeny were the same as that of CLd. CLd-AXE amoebae grew less well on lawns of bacteria than did CLd-BACT amoebae; however, the good growth of the progeny strain, LU310, showed that poor growth on bacteria is not necessarily a characteristic of strains able to grow in axenic medium.

Axenic culture will be useful for obtaining large numbers of cells in exponential growth for mutagenesis. It is still not possible to clone amoebae on axenic agar media for isolation of mutants (unpublished results). However, since the tests of CLd-AXE indicate that rapid reversion of the axe mutations does not occur during culture on bacteria, it should be possible to isolate in the presence of bacteria mutants which retain the ability to grow axenically. One disadvantage of using CLd-AXE for mutant isolation might be its low plating efficiency on FK/LIA, but the greatly improved growth and plating efficiency of clone LU310 indicates that this problem may be overcome by using an appropriate strain. Since axenic cultures cannot be established from inccula of less than $5 \times 10^{5} \mathrm{cells}^{-1}$, clones would first have to be cultured on bacteria and could not be inoculated into liquid culture by transfer of a few cells from a single plaque. However, preliminary evidence suggests that this problem may be alleviated by the use of growth-conditioned medium.

Crosses between CLd-AXE amoebae and amoebae of known genotype can be used to construct a range of strains carrying known genetic markers and able to grow in axenic medium. A long period of 'adaptation' in axenic medium will therefore not be necessary before mutant strains can be tested for the expression of mutations identified in plasmodia. With temperature sensitive mutations, it is particularly useful that amoebae and plasmodia can be compared in the same semi-defined medium and at the same non-permissive temperature. Previous tests on E27, for example, had indicated that the amoebae were not temperature sensitive at $31^{\circ} \mathrm{C}$ on $E$. coli; but growth of CLd is also poor under these conditions (Gingold et al., 1976 and unpublished observations). Although at present the chemically defined media used for plasmodia and amoebae are different, it is possible to test for specific nutritional requirements in both stages of the life-cycle.

The financial support of the SRC (grant no. B/RG89505) is gratefully acknowledged and we thank Mr T. G. Burland for providing isolated duck erythrocyte nuclei.

\section{REFERENCES}

Adler, P. N. \& Holt, C. E. (1974). Changes in properties of Physarum polycephalum amoebae during extended culture. Journal of Bacteriology 120, 532-533.

Anderson, R. W. \& Dee, J. (1977). Isolation and analysis of amoebal-plasmodial transition mutants in the myxomycete Physarum polycephalum. Genetical Research 29, 21-34.

COOKE, D. J. \& DEE, J. (1974). Plasmodium formation without change in nuclear DNA content in Physarum polycephalum. Genetical Research 23, 307-317.

Cooke, D. J. \& DeE, J. (1975). Methods for the isolation and analysis of plasmodial mutants in Physarum polycephalum. Genetical Research 24, 175-187.

DEE, J. (1966). Multiple alleles and other factors affecting plasmodium formation in the true slime mould Physarum polycephalum Schw. Journal of Protozoology 13, 610-616.

DeE, J. \& Poulter, R. T. M. (1970). A gene conferring actidione resistance and abnormal mor- phology on Physarum polycephalum plasmodia. Genetical Research 15, 35-41.

Dee, J., Wheals, A. E. \& Holt, C. E. (1973). Inheritance of plasmodial valine requirement in Physarum polycephalum. Genetical Research 21, 87-101.

Gingold, E. C., Grant, W. D., Wheals, A. E. \& WREN, M. (1976). Temperature sensitive mutants of the slime mould Physarum polycephalum. II. Mutants of the plasmodial phase. Molecular and General Genetics 149, 115-119.

Goodman, E. M. (1972). Axenic culture of myxamoebae of the myxomycete Physarum polycepkalum. Journal of Bacteriology 111, 242-247.

HAUGLI, F. B. (1971). Mutagenesis, selection and genetic analysis in Physarum polycephalum. Ph.D. thesis, University of Wisconsin, U.S.A.

HenNey, H. R. \& HenNey, M. R. (1968). Nutritional requirements for the growth in pure culture of the myxomycete Physarum rigidum and related species. Journal of General Microbiology 53, 333-339. 
McCullough, C. H. R., Cooke, D.J., Foxon, J. L., Sudbery, P. E. \& Grant, W. D. (1973). Nuclear DNA content and senescence in Physarum polycephalum. Nature New Biology 245, 263-265.

McCullough, C. H. R. \& DeE, J. (1976). Defined and semi-defined media for the growth of amoebae of Physarum polycephalum. Journal of General Microbiology 95, 151-158.

Mirsky, A. E. \& Ris, H. (1951). The desoxyribonucleic acid content of animal cells and its evolutionary significance. Journal of General Physiology 34, 451-462.

MoHBERG, J. \& Rusch, H. P. (1971). Isolation and DNA content of nuclei of Physarum polycephalum. Experimental Cell Research 66, 305-316.

MoHBerg, J. (1977). Nuclear DNA content and chromosome numbers throughout the life cycle of the Colonia strain of the myxomycete Physarum polycephalum. Journal of Cell Science 24, 95-108.

Poulter, R. T. M. \& DEe, J. (1968). Segregation of factors controlling fusion between plasmodia of the slime mould Physarum polycephalum. Genetical Research 12, 71-79.

WheAls, A. E. (1970). A homothallic strain of the myxomycete Physarum polycephalum. Genetics 66, 623-633.

Williams, K. L., Kessin, R. H. \& Newell, P. C. (1974). Parasexual genetics in Dictyostelium discoideum: mitotic analysis of acriflavin resistance and growth in axenic medium. Journal of General Microbiology 84, 59-69. 